\title{
Fusion of Total Variation Filter and Weighted Bilateral Filter in Image Denoising
}

\author{
Rashid $\mathrm{Ali}^{1}$, Peng Yunfeng ${ }^{1}$ and Suman Kumari Gupta ${ }^{2}$ \\ ${ }^{1}$ School of Computer \& Communication Engineering, University of Science and \\ Technology Beijing (USTB), No. 30 Xueyuan Road, Beijing, 100083 China \\ 710072, China \\ ${ }^{2}$ Department of IT, Islington College (London Metropolitan University), \\ Kathmandu, Nepal \\ Iist_rashid89@yahoo.com,
}

\begin{abstract}
Image denoising is an established issue in signal processing. The most usual noise reduction methods use the average or linear methods such as wiener filtering noise reduction but the result and visual effect is not ideal. This article aims to propose an image denoising algorithm by combining two well-known image denoising techniques: i.e., total variation filter (TVF) and weighted bilateral filter (WBF), to obtain an improved image denoising result as compared to individual filters. We have used standard images data sets for our experiment. The results clearly show that the proposed algorithm results in a better quality image, both in term of numerically and visually. The image denoising results are compared quantitatively in terms of peak signal to noise ratio (PSNR) and it can be seen that the combination of total variation filter and weighted bilateral filter results in an improved image as compare to only total variation filter or only weighted bilateral filter. The proposed research has future implications as proposed scheme is applied on standard images but it can be used in real time image denoising with some modifications.
\end{abstract}

Keywords: Image denoising, Total variation filter, Weighted bilateral filter, PSNR. WGN, AWGN. Weighted function, Bilateral filter

\section{Introduction}

Due to different kinds of interference factors existing in the formation transmission process, the actual image may be polluted by noise. Noise reduction from images is very active research topic and has application in vast areas such as edge detection, image fusion, computer vision processing, image segmentation, pattern recognition and feature extraction [1]. The purpose is to develop the image peak signal to noise ratio (PSNR) large, in addition to improve image worth and highlight features of the image itself. Image denoising is essentially the use of noise signal and the energy of image signal having different distribution in frequency domain [2]. The image energy is normally dispersed in the low frequency region [3]. The image detailed energy is dispersed in the high frequency range [4], although the noise energy is generally focused in the high frequency region [5]. So from the aspect of frequency, high frequency noise sign can be separated effectively from the image.

Image denoising is an established issue in signal processing [6]. The most usual noise reduction methods use the average or linear methods such as wiener filtering noise reduction but the result and visual effect is not ideal [7]. The superior skill in time frequency analysis of wavelet transform is paid more and more attention in the field of

Received (November 17, 2017), Review Result (January 17, 2018), Accepted (February 1, 2018) 
image denoising [8], creating a new nonlinear noise reduction method from the ground and hence it achieved good results.

Then again, image denoising from natural and unnatural images is in the not too distant past a testing issue in image processing. Undoubtedly, wavelets transform based methodologies have proficient noise diminishing utmost in photographic images $[9,10,11]$ and promising results are represented in these references. Starting late, multiple wavelets commence image denoising methodologies are moreover reported with outstanding execution [12,13]. Regardless, still these strategies have issues on a significant noisy framework [14] Also, wavelet based techniques are computationally costly and are not appropriate for non-characteristic images $[15,16]$.

In this paper a new and an efficient algorithm is proposed using combination of total variation filter (TVF) and weighted bilateral filters (WBF). Both techniques are presented in detail and are combined for enhanced image denoising using standard images. Results of individual techniques are also compared with combined technique that also reflects the efficiency of the proposed algorithm. In short, proposed algorithm results in images with:

- Improved reconstruction of sharp edges.

- Improved reconstruction of repeating patterns.

- Improved preservation of contrast.

- Improved PSNR results.

\section{Total Variation Filter (TVF)}

The total variation (TV) of a flag measures how much the flag changes between flag qualities. In particular, the total variation of an $\mathrm{N}$ point signal $x(n), 1 \leq n \leq N$ is characterized as (1).

$$
T V(x)=\sum_{n=2}^{N}|x(n)-x(n-1)|
$$

The total variation of $x$ can likewise be composed as:

$$
T V(x)=\|D x\|_{1}
$$

Where,

$$
D=\left[\begin{array}{ccccc}
-1 & 1 & & & \\
& -1 & 1 & & \\
& & & \ldots . & \\
& & & -1 & 1
\end{array}\right]
$$

is a grid of size $(N-1) \times N$. We expect we watch the sign $x$ debased by additive white gaussian noise (AWGN), $y=x+n \quad y, x, n \in \mathrm{R} h$.

One way to deal with assessment $x$ is to locate the sign $x$ minimizing the target capacity

$$
J(x)=\|y-x\|_{2}^{2}+\lambda\|D x\|_{1}
$$

This methodology is called total variation filter (TVF) denoising. The regularization parameter $\lambda$, which controls the amount of smoothing is also performed. Bigger noise level calls for bigger $\lambda$.

\subsection{Algorithm for Total Variation Denoising (TVF)}

We will expect a more broad type of the target capacity: 


$$
J(x)=\|y-x\|_{2}^{2}+\lambda\|A x\|_{1}
$$

In above equation $x$ is vector, where $A$ is a framework of size $\mathrm{M} \times \mathrm{N}$. The optimal estimation of the target capacity is indicated as:

$$
J_{*}=\min _{x}\|y-x\|_{2}^{2}+\lambda\|A x\|_{1}
$$

The minimization of this target capacity is entangled by the way that the $l l$ standard is note differentiable. In this manner, a way to deal with minimize $\mathrm{J}(\underline{x})$ is to utilize the double plan. To determine the double plan, take note of that the outright estimation of a scalar $x$ can be composed in the accompanying winding structure:

$$
|x|=\max _{|z| \leq 1} z x
$$

In above equation $x$ is scalar. The upside of this structure is that the nonlinearity of the capacity is exchanged to the plausible set. In like manner, note that the $l l$ standard of a vector $x$ can be composed as:

$$
\|x\|_{1}=\max _{|z| \leq 1} z^{t} x
$$

Where the condition $|z| \leq 1$ is taken component insightful. So also,

$$
\|A x\|_{1}=\max _{|z| \leq 1} z^{t} A x
$$

Thusly, we can compose the target capacity $J(x)$ in mathematical statement (5) as

$$
J(x)=\|y-x\|_{2}^{2}+\lambda \max _{|z| \leq 1} z^{t} A x
$$

Or

$$
J(x)=\max _{|z| \leq 1}\|y-x\|_{2}^{2}+\lambda z^{t} A x .
$$

The optimal estimation of the target capacity in comparison (7) is

$$
J_{*}=\min _{x} \max _{|z| \leq 1}\|y-x\|_{2}^{2}+\lambda z^{t} A x
$$

We need to discover the vector $x$ giving this worth, yet it will be helpful to discover both $x$ and the auxiliary $z$, characterizing

$$
F(x, z):=\|y-x\|_{2}^{2}+\lambda z^{t} A x
$$

We can compose

$$
J_{*}=\min _{x} \max _{|z| \leq 1} F(x, z)
$$

Since $F(x, z)$ is convex in $x$ and concave in $z$, the optimal worth $J$ is a saddle purpose of $F(x, z)$. By the min-max property, we can compose 


$$
J_{*}=\max _{|z| \leq 1} \min _{x} F(x, z)
$$

Or

$$
J_{*}=\max _{|z| \leq 1} \min _{x}\|y-x\|_{2}^{2}+\lambda z^{t} A x
$$

Which is the double plan of the TVF denoising issue. The internal minimization issue in (17) can be comprehended as takes after:

$$
\frac{\partial}{\partial x} F(x, z)=-2(y-x)+\lambda A^{t} z
$$

so

$$
\frac{\partial}{\partial x} F(x, z)=0 \Rightarrow x=y-\frac{\lambda}{2} A^{t} z
$$

Substituting (19) once more into (17) gives

$$
J_{*}=\max _{|z| \leq 1}\left\|\frac{\lambda}{2} A^{t} z\right\|_{2}^{2}+\lambda z^{t} A\left(y-\frac{\lambda}{2} A^{t} z\right)
$$

In the wake of rearranging we have

$$
J_{*}=\max _{|z| \leq 1}-\frac{\lambda^{2}}{4} z^{t} A A^{t} z+\lambda z^{t} A y
$$

On the other hand equally, the minimization issue

$$
z_{*}=\underset{|z| \leq 1}{\arg \min } z^{t} A A^{t} z-\frac{4}{\lambda} z^{t} A y
$$

Setting the subsidiary concerning $z$ as zero gives $t$ comparison

$$
A A^{t} z=\frac{2}{\lambda} A y
$$

Which requires the answer for a possibly huge arrangement of straight mathematical statements and moreover does not yield an answer $z$ fulfilling the imperative $|z| \leq 1$. To discover $Z$ taking care of the obliged minimization issue, the majorization-minimization (MM) strategy can be utilized characterizing.

$$
D(z)=z^{t} A A^{t} z-\frac{4}{\lambda} z^{t} A y
$$

What's more, setting $\mathrm{z}^{(\mathrm{i})}$ as purpose of fortuitous event, we can discover distinct majorizer of $D(z)$ by including the non-negative capacity

$$
\left(z-z^{(i)}\right)^{t}\left(\alpha I-A A^{t}\right)\left(z-z^{(i)}\right)
$$

To $D(z)$, where $\alpha$ is more noteworthy than or equivalent to the most extreme eigen value of $A A^{t}$. So a majorizer of $D(z)$ is given by 


$$
D(z)+\left(z-z^{(i)}\right)^{t}\left(\alpha I-A A^{t}\right)\left(z-z^{(i)}\right)
$$

What's more, utilizing the MM approach, the overhaul mathematical statement for $z$ is given by:

$$
\begin{aligned}
& z^{(i+1)}=\underset{|z| \leq 1}{\arg \min } D(z)+\left(z-z^{(i)}\right)^{t}\left(\alpha I-A A^{t}\right)\left(z-z^{(i)}\right) \\
& z^{(i+1)}=\underset{|z| \leq 1}{\arg \min } \alpha z^{t} z-2\left(A\left(\frac{2}{\lambda} y-A^{t} z^{(i)}\right)+\alpha z^{(i)}\right)^{t} z+K \\
& =\underset{|z| \leq 1}{\arg \min } z^{t} z-2\left(\frac{1}{\alpha} A\left(\frac{2}{\lambda} y-A^{t} z^{(i)}\right)+z^{(i)}\right)^{t} z \\
& =\arg \min z^{t} z-2 b^{t} z
\end{aligned}
$$

where

$$
b=z^{(i)}+\frac{1}{\alpha} A\left(\frac{2}{\lambda} y-A^{t} z^{(i)}\right)
$$

We have to discover $z \in \mathrm{R}^{M}$ minimizing $z^{t} z-2 b t z$ subject to the requirement $|z| \leq 1$. Consider first the scalar case:

$$
\underset{|z| \leq 1}{\arg \min } z^{2}-2 b z
$$

The arrangement is given by cut-out capacity:

$$
\operatorname{clip}(b, T):=\left\{\begin{array}{cl}
b & |b| \leq T \\
T \operatorname{sign}(b) & |b| \geq T
\end{array}\right.
$$

Note that the vector case (27) is distinct the components of $z$ are uncoupled so the compelled minimization can be performed component insightful. Along these lines, an upgrade comparison for $z$ is given by:

$$
z^{(i+1)}=\operatorname{clip}\left(z^{(i)}+\frac{1}{\alpha} A\left(\frac{2}{\lambda} y-A^{t} z^{(i)}\right), 1\right)
$$

Where $i$ is the iteration index, In rundown, the determined iterative calculation for TVF denoising comprises of an instatement of $z$, say $z^{(0)}=0$, the redesign comparison (33). When the upgrade has joined agreeable to one, the denoised signal $x$ is given by (18). We call this the "iterative clipping algorithm". The iterative section calculation for TVF denoising can likewise be composed as

$$
x^{(i+1)}=y-\frac{\lambda}{2} A^{t} z^{(i)}
$$




$$
z^{(i+1)}=\operatorname{clip}\left(z^{(i)}+\frac{2}{\alpha \lambda} A x^{(i+1)}, 1\right)
$$

Scaling $\mathrm{z}$ by $\lambda / 2$, we have the following equivalent form:

$$
\begin{aligned}
& x^{(i+1)}=y-A^{t} z^{(i)} \\
& z^{(i+1)}=\operatorname{clip}\left(z^{(i)}+\frac{1}{\alpha} A x^{(i+1)}, \frac{\lambda}{2}\right)
\end{aligned}
$$

\section{Weighted Bilateral Filter (WBF)}

\subsection{Bilateral Filter (BF)}

Bilateral Filter is used to smooth images with the benefit of saving edges, by methods for figuring the yield at a pixel as a weighted average of the relating pixels in the region chose by the filter mask. The coefficient is controlled by geometric closeness and shading esteem likeness amongst pixels, and pixels that are like the middle pixel in both area and range will have higher weights [18].

The motivation behind why the bilateral filter can smooth the commotion while keeping edges in place is as per the following. At an edge pixel, the bilateral filter turns into a lengthened gaussian filter that is arranged along the edge by consolidating the range filter and the domain filter, which guarantees that pixels averaging is done generally along the edge and is incredibly diminished in the inclination heading. Because of this decent property, bilateral filter is broadly utilized for sharpness enhancement and commotion evacuation [19]. The coefficient is given by

$$
W_{B}=W_{s} * W_{R}
$$

Where

$$
W S=e^{-\frac{|x-y|^{2}}{2 \sigma_{s}^{2}}}
$$

and

$$
W_{R}=e^{-\frac{\left|I_{x}-I_{y}\right|^{2}}{2 \sigma_{s}^{2}}}
$$

Nonetheless, it has been broadly seen that bilateral filter isn't fit for a wide range of noise. It has a decent execution for gaussian noise, while it can't evacuate drive noise [20]. At the point when pixels in filter mask are contaminated by the motivation noise, bilateral filter can't recognize them and evacuate them, which make the weighted average insecure. The reason is that pixel contaminated by the drive noise is typically not comparative with pixels in the neighborhood, which influences these pixels to be mixed up as the edge of the image and will be preserved.

\subsection{Weighted Bilateral Filter (WBF)}

The weighted bilateral filter (WBF) can execute as a detail data protecting smoothing and gaussian noise expelling filter like the bilateral filter (BF), derived from the bilateral 
filter, the coefficient of weighted bilateral filter (WBF) is consolidated by geometric closeness and shading esteem similitude among the pixels, and a weighted coefficient which is dictated by whether the pixel is contaminated by noise.

For "detect and replace" or "decision-based" strategy, which is the most generally utilized motivation noise expulsion procedures [21], needs two stages to finish its capacity, weighted bilateral filter (WBF) can act naturally versatile to expel driving forces while being able to smooth gaussian noise, which relies upon a weighted coefficient. weighted bilateral filter (WBF) is gotten from the bilateral filter, and the coefficient of weighted bilateral filter (WBF) is joined by the coefficient of bilateral filter, and a weighted coefficient. The weighted coefficient is dictated by whether the pixel is contaminated by motivation noise. The configuration of weighted bilateral filters (WBF) coefficient is as per the following:

$$
W=W_{N} * W_{B}=W_{N} * W_{S} * W_{R}
$$

$W_{N}$ speaks to the weighted coefficient of weighted bilateral filter (WBF), and its esteem decides if the pixel will be expelled or be safeguarded. In the event that the pixel has a major contrast with different pixels, it implies that the pixel is dirtied by drive commotion and $W_{N}$ approaches zero, which implies the pixel will be expelled. Else, it will approach one, which implies it will be protected. That implies if the pixel is contaminated, it won't be considered in the operation, or else it fills in as a bilateral filter.

We can pick a capacity which has property as above said to accomplish it. In weighted bilateral filter (WBF) utilize the accompanying capacity:

$$
W_{1}=1-\prod_{\substack{y \neq x \\ y \in \text { window-shape }}}\left(1-e^{-\frac{\mid\left\langle a-\left.I_{b}\right|^{2}\right.}{2 \sigma_{n}^{2}}}\right)
$$

To acknowledge what we need. The window shape relies upon the execution you need to accomplish as per the intensity of impulse noise. For instance, if the intensity is low, we can assume that the likelihood when more than one pixel is contaminated in a window is low and $W_{N}$ can be set as:

$$
W_{N}=1-\prod_{\substack{y \neq x \\ y \in \Omega}}\left(1-e^{-\frac{\left(\left(a-t_{b}\right)^{2}\right.}{2 \sigma_{n}^{2}}}\right)
$$

Where $\Omega$ speaks to the square window, as $3 \times 3$. On the off chance that the intensity is higher, we can utilize a more mind boggling window shape to enhance the capacity of perceiving impulse noise, for example:

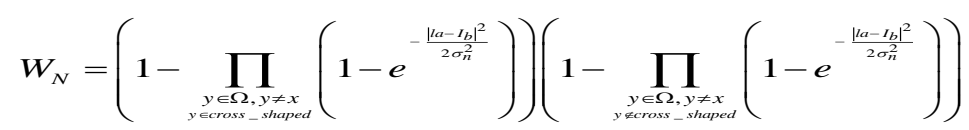

This implies if the probability that more than two pixels is dirtied is lower, at that point the other adulterated pixel must be in cross shaped window or in the rest window, subsequently the coefficient patterns to be zero, which implies that this clamor point is expelled and the yield is a weighted average of the encompassing pixels remaining. Else, you can pick other sort of window shape you need to have diverse execution.

The execution of weighted bilateral filter (WBF) relies upon these parameters $\sigma_{\mathrm{n}}, \sigma_{\mathrm{s}}$ and $\sigma_{\mathrm{r} .} \sigma_{\mathrm{s}}$ and $\sigma_{\mathrm{r}}$ is the same as in bilateral filter $(\mathrm{BF})$. From the property of the weighted coefficient we can get that, if the fact of the matter is spotless or is recently contaminated 
by gaussian noise, at that point the filter acts as a bilateral filter (BF), or else the dirtied pixel will be expelled. As a rule, the weighted bilateral filter (WBF) has a decent execution in expelling drive noise without yielding the capacity of bilateral filter (BF) to safeguard detail data.

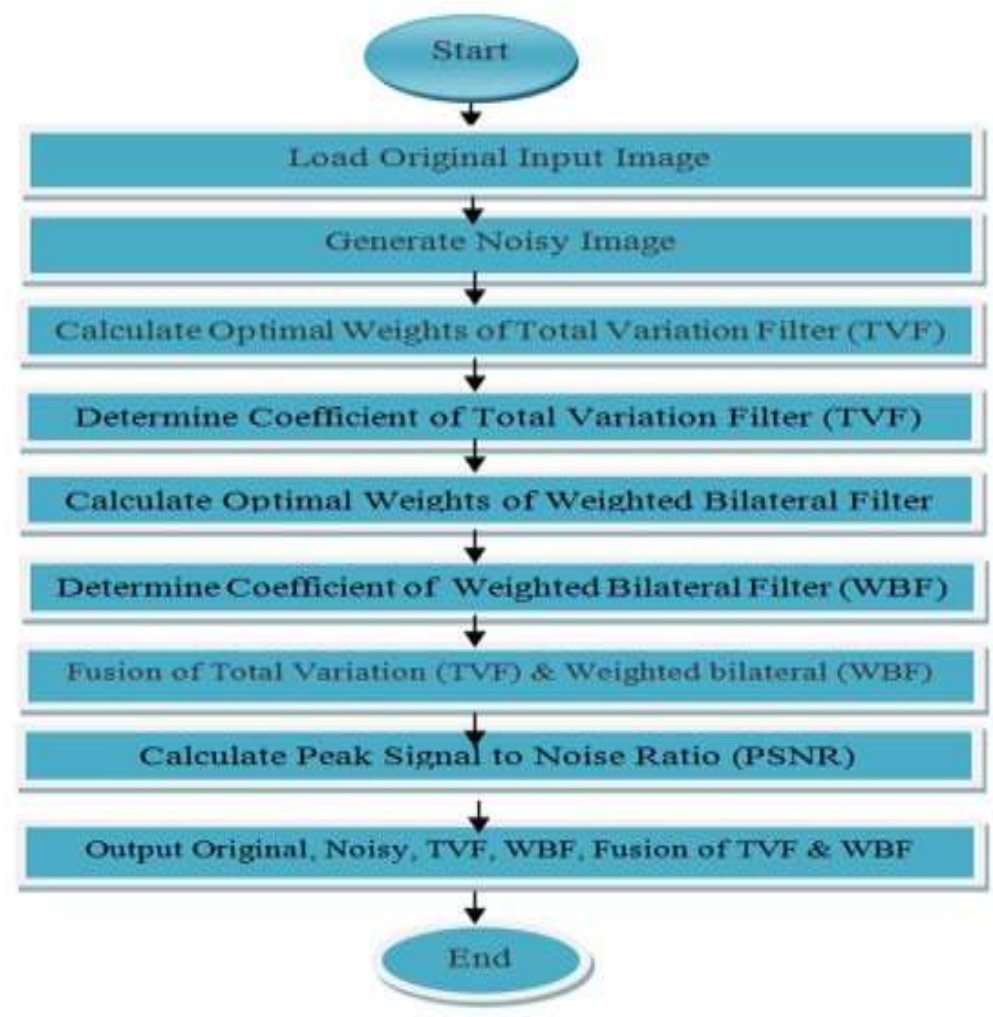

Flow Chart: Proposed Algorithm Sequence

\section{Performance Measures}

This section presents the implementation output of total variation filter (TVF) and weighted bilateral filter (WBF). The proposed algorithm is implemented on set of images and peak signal to noise ratio (PSNR) for each of these outputs is computed. It is worth mentioning here that by far there is no criterion of image quality evaluation that can be accepted generally by all. Peak signal to noise ratio (PSNR) is one of the criteria which is used to evaluate the quality of images and has been used in this work. The investigations are performed on a set of gray scale images of size 512x512 Einstein and 256x256 House in MATLAB stage. In our experiment we used white gaussian noise (WGN) and additive white gaussian noise (AWGN) to check the feasibility of this proposed method and we got better result for both noises at different noised levels.

There is no generally acknowledged image denoising quality estimation. In this manner, we think about our denoised images both objectively and subjectively. For target estimation or numerical estimation, we display the broadly utilized peak signal to noise ratio (PSNR) for each watched image, as there is no exact guideline to choose one measure over the other.

Assessment of the subjective quality is finished by contrasting parts of images one next to the other. In visual assessment we especially analyze sharpness of edges and safeguarding of complexity. 


\section{Output Results}

Different standards images were used for experiments. Original Einstein and House images can be seen in Figure $1 \&$ 10. Noisy images using noise level 10, 20, and 30 can be seen in Figure $2 \& 11$. The denoised image using total variation filter (TVF) can be seen in Figure $3 \&$ 12. Denoised image using weighted bilateral filter (WBF) can be seen in Figure $4 \& 13$. Improved denoised image using total variation filter (TVF) and weighted bilateral filter (WBF) filter can be seen in Figure $5 \& 14$.

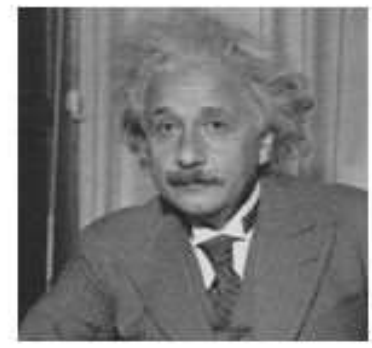

Figure 1. Einstein images (512×512)
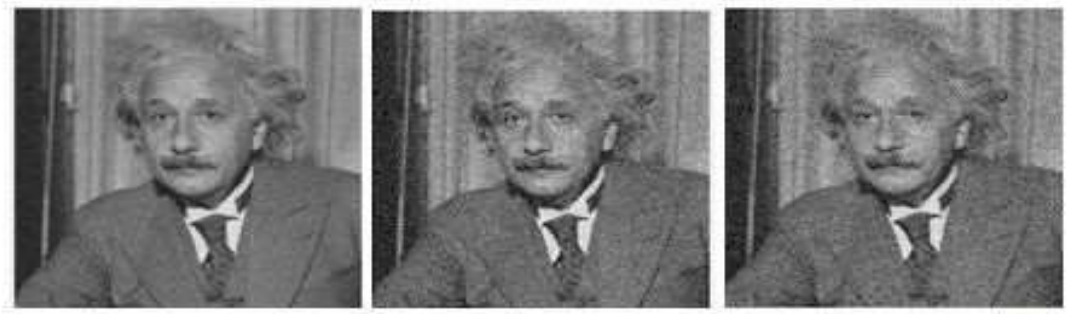

Noisy image $\sigma=10$ Noisy image $\sigma=20$ Noisy image $\sigma=30$

Figure 2. Einstein noisy images
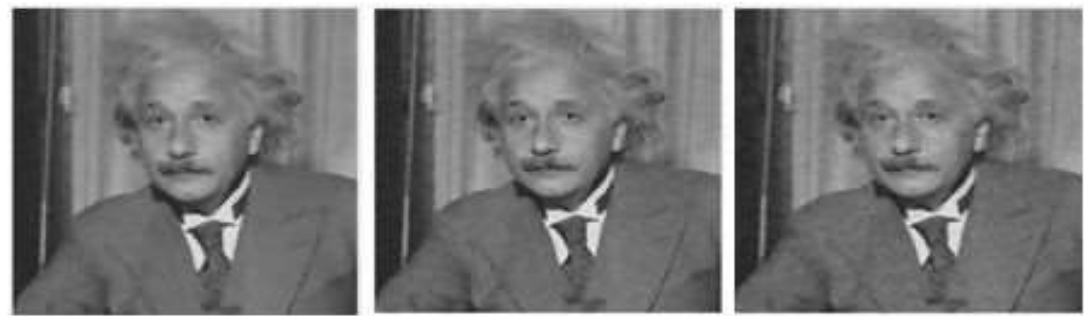

\section{Denoised PSNR 32.34 Denoised PSNR 31.93 Denoised PSNR 30.87} Figure 3. Einstein TVF denoised images [19]
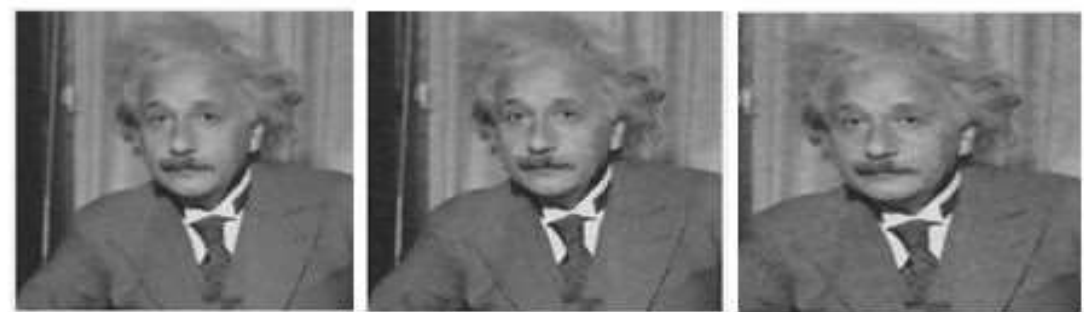

Denoised PSNR 33.17Denoised PSNR 32.08 Denoised PSNR 30.89 Figure 4. Einstein WBF denoised images [22] 

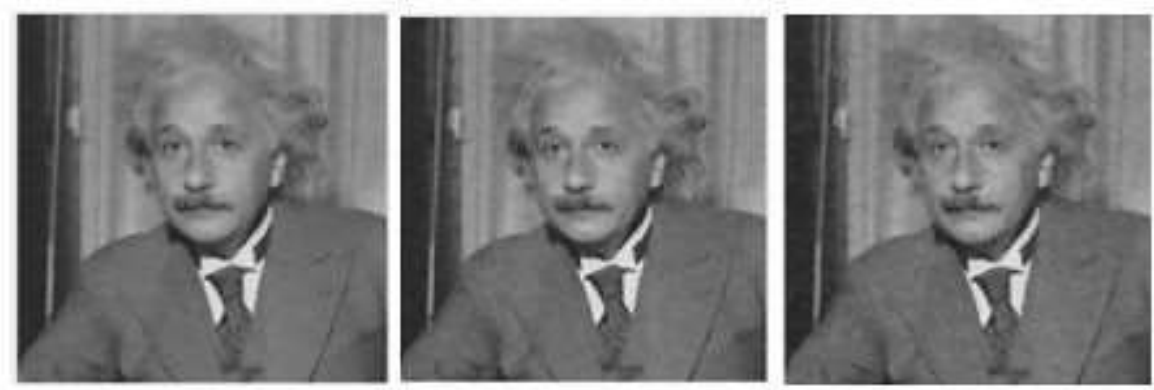

\section{Denoised PSNR 33.28 Denoised PSNR 32.12 Denoised PSNR 30.94 Figure 5. Combination of TVF and WBF denoised images (Proposed approach)}

Noisy images using noise level 40, and 50 can be seen in Figure $6 \& 15$. The denoised image using TVF filter can be seen in Figure $7 \& 16$. The denoised image using WBF filter can be seen in Figure $8 \&$ 17. Improved denoised image using TVF and WBF filter can be seen in Figure $9 \& 18$.
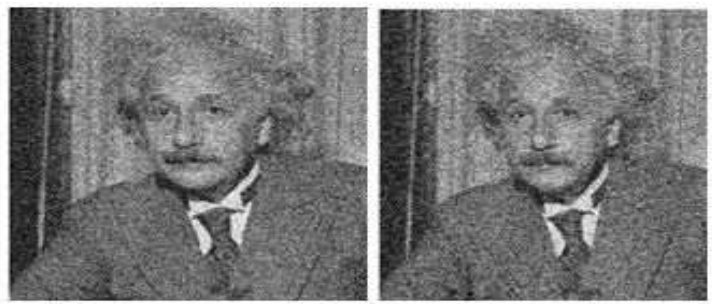

Noisy image $\sigma=40$ Noisy image $\sigma=50$ Figure 6. Einstein noisy images
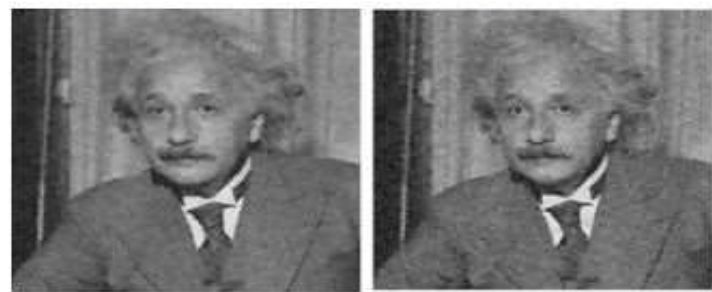

\section{Denoised image PSNR 29.20 Denoised image PSNR 27.22} Figure 7. Einstein TVF Denoised images [19]
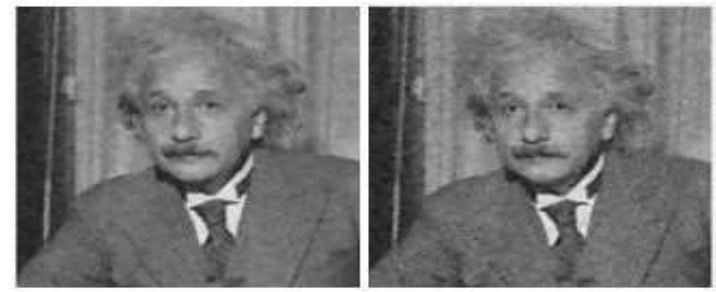

Denoised image PSNR 29.22 Denoised image PSNR 27.25

Figure 8. Einstein WBF denoised images [22]

Figure 1 to 9 presents subjective comparison of denoising performance between TVF, WBF image and combination of TVF and WBF for standard Einstein images noise level $\sigma=10,20,30,40$ and 50 . 


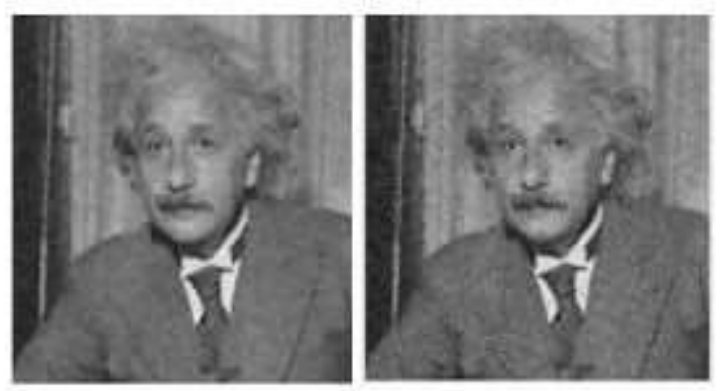

Denoised image PSNR 29.32 Denoised image PSNR 27.39 Figure 9. Combination of TVF and WBF denoised images (Proposed approach)

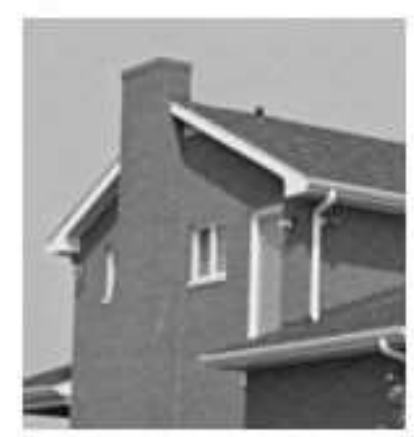

Figure 10 . House images $(256 \times 256)$
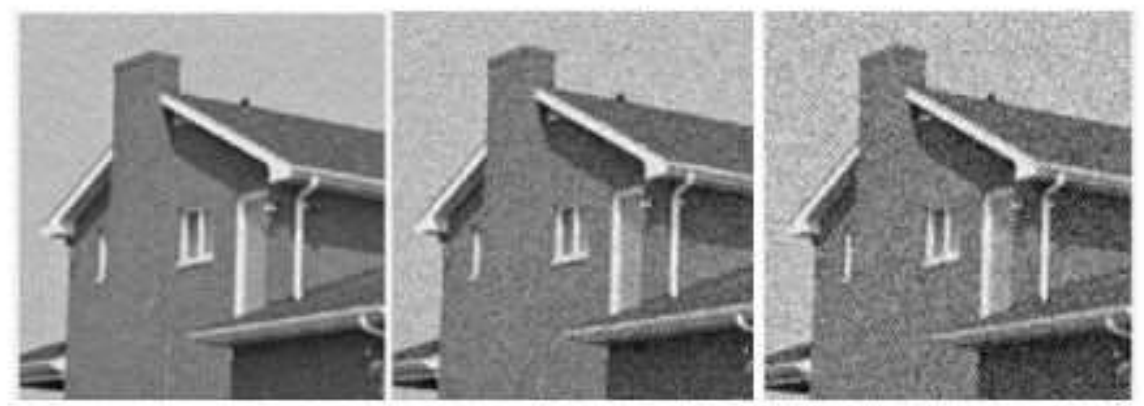

Noisy image $\sigma=10$ Noisy image $\sigma=20$ Noisy image $\sigma=\mathbf{3 0}$

Figure 11. House noisy images
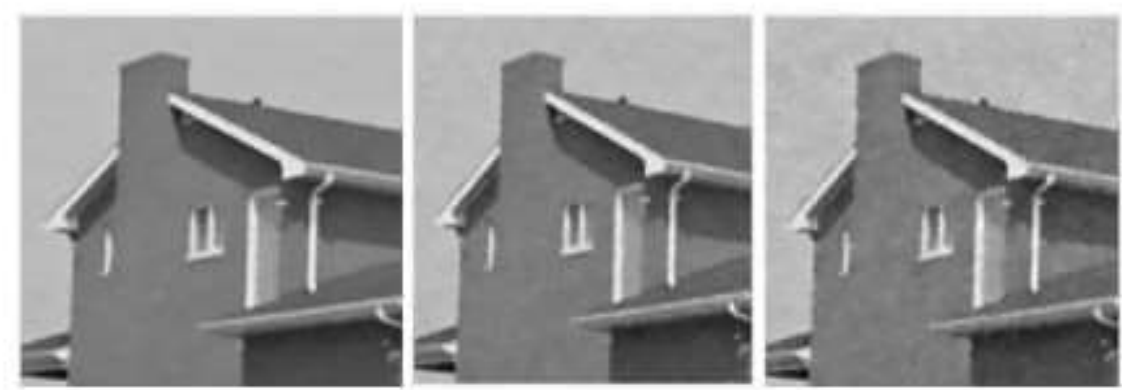

Denoised PSNR 31.67 Denoised PSNR 30.98 Denoised PSNR 29.69

Figure 12. House TVF denoised images [19] 

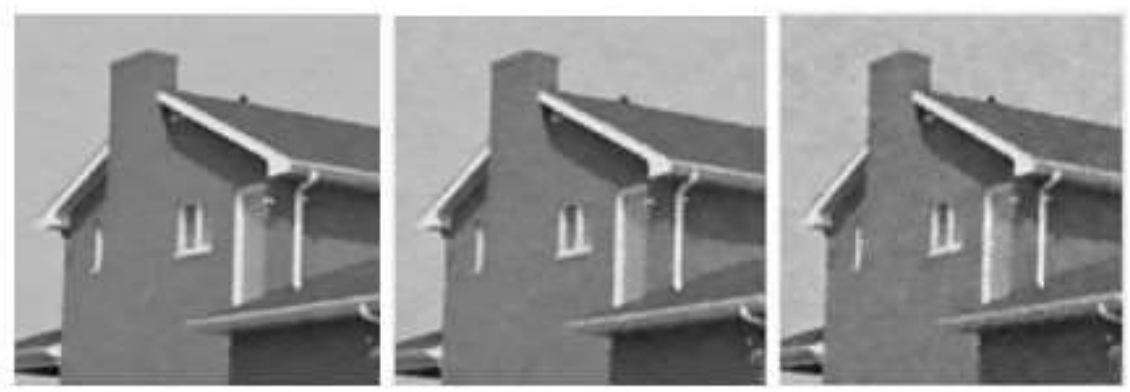

Denoised PSNR 33.53 Denoised PSNR 31.30 Denoised PSNR 29.74

Figure 13. House WBF denoised images [22]
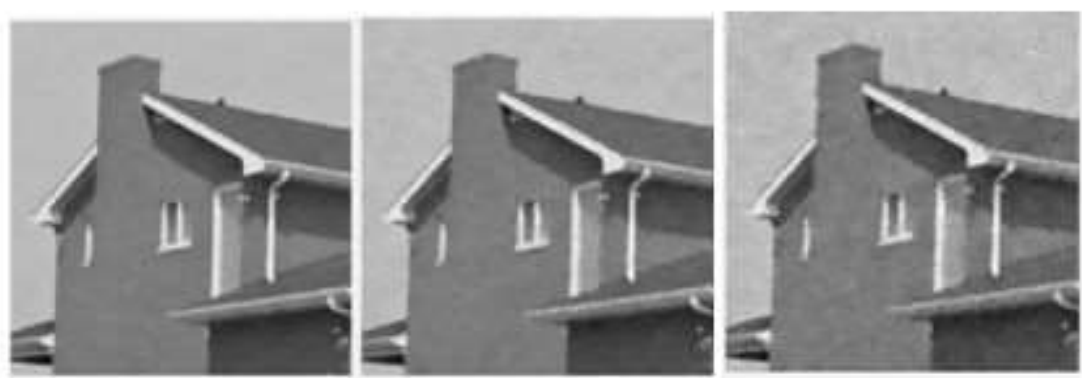

Denoised PSNR 33.66 Denoised PSNR 31.37 Denoised PSNR 29.79 Figure 14. Combination of TVF and WBF denoised images (Proposed approach)
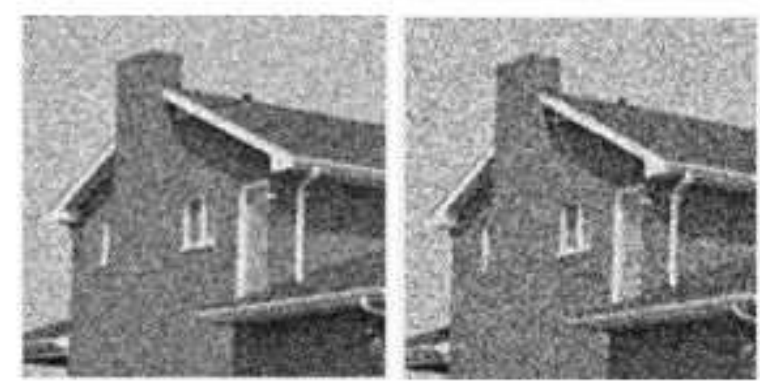

Noisy image $\sigma=40$ Noisy image $\sigma=50$

Figure 15. House noisy images
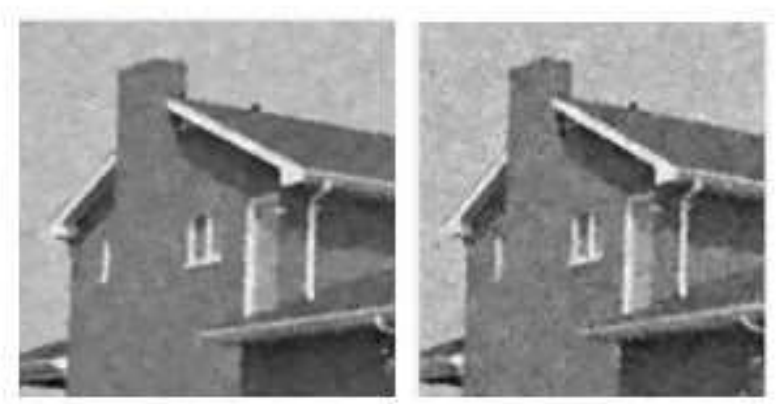

Denoised image PSNR 28.18 Denoised image PSNR 26.35

Figure 16. House TVF denoised images [19] 

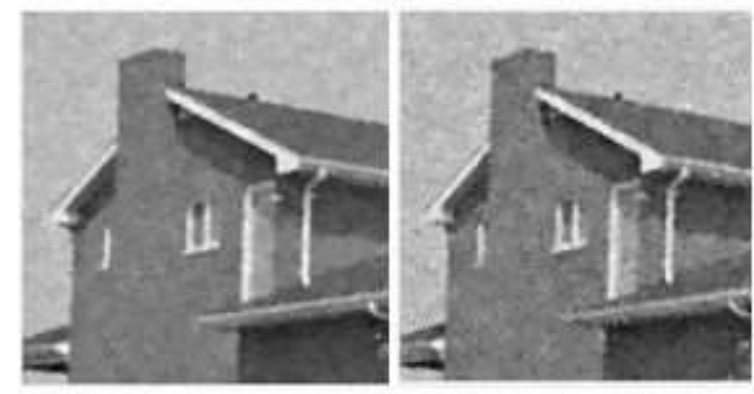

Denoised image PSNR 28.20 Denoised image PSNR 23.36

Figure 17. House WBF denoised images [22]
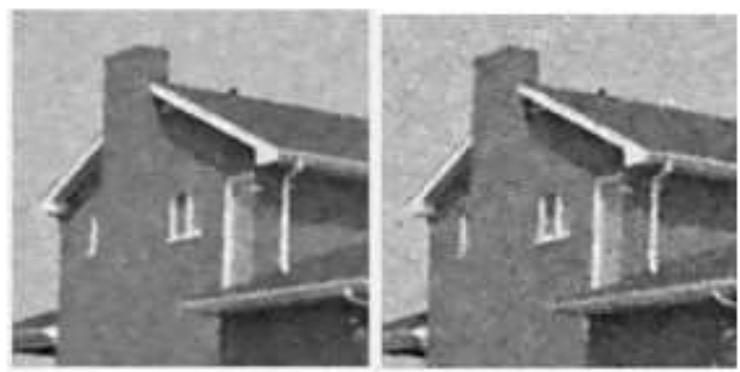

Denoised image PSNR 28.27 Denoised image PSNR 26.46

Figure 18. Combination of TVF and WBF denoised images (Proposed approach)

The above Figure 10 to 18 shows subjective comparison of denoising performance between TVF, WBF Image and combination of TVF and WBF for standard test image House at noise level $\sigma=10,20,30,40$ and 50 .

\section{Table 1. Comparison of PSNR on Different Noise Levels on Einstein Image}

\begin{tabular}{|c|c|c|c|c|c|}
\hline \multirow{6}{*}{ Image Name } & $\begin{array}{c}\text { Noise Level } \\
\sigma\end{array}$ & $\begin{array}{c}\text { Noisy Image } \\
\text { (PSNR) }\end{array}$ & $\begin{array}{c}\text { Denoised } \\
\text { Image } \\
\text { (PSNR) } \\
\text { using TVF }\end{array}$ & $\begin{array}{c}\text { Denoised Image } \\
\text { (PSNR) } \\
\text { using WBF }\end{array}$ & $\begin{array}{c}\text { Combination of } \\
\text { TVF and WBF } \\
\text { (PSNR) } \\
\text { (Proposed } \\
\text { approach) }\end{array}$ \\
\hline \multirow{4}{*}{ Einstein } & 10 & 28.13 & 32.34 & 33.17 & $\mathbf{3 3 . 2 8}$ \\
\cline { 2 - 6 } & 20 & 22.11 & 31.93 & 32.08 & $\mathbf{3 2 . 1 2}$ \\
\cline { 2 - 6 } & 30 & 18.59 & 30.87 & 30.89 & $\mathbf{3 0 . 9 4}$ \\
\cline { 2 - 6 } & 40 & 16.06 & 29.20 & 29.22 & $\mathbf{2 9 . 3 2}$ \\
\cline { 2 - 6 } & 50 & 14.17 & 27.22 & 27.25 & $\mathbf{2 7 . 3 9}$ \\
\hline
\end{tabular}

Table 2. Comparison of PSNR on Different Noise Levels on House Image

\begin{tabular}{|c|c|c|c|c|c|}
\hline \multirow{5}{*}{ Image Name } & $\begin{array}{c}\text { Noise Level } \\
\sigma\end{array}$ & $\begin{array}{c}\text { Noisy Image } \\
\text { (PSNR) }\end{array}$ & $\begin{array}{c}\text { Denoised } \\
\text { Image } \\
\text { (PSNR) } \\
\text { using TVF }\end{array}$ & $\begin{array}{c}\text { Denoised Image } \\
\text { (PSNR) } \\
\text { using WBF }\end{array}$ & $\begin{array}{c}\text { Combination of } \\
\text { TVF and WBF } \\
\text { (PSNR) } \\
\text { (Proposed } \\
\text { approach) }\end{array}$ \\
\hline \multirow{4}{*}{ House } & 10 & 28.14 & 31.67 & 33.53 & $\mathbf{3 3 . 6 6}$ \\
\cline { 2 - 6 } & 20 & 22.09 & 30.98 & 31.30 & $\mathbf{3 1 . 3 7}$ \\
\cline { 2 - 6 } & 30 & 18.60 & 29.69 & 29.74 & $\mathbf{2 9 . 7 9}$ \\
\cline { 2 - 6 } & 40 & 16.08 & 28.18 & 28.20 & $\mathbf{2 8 . 2 7}$ \\
\cline { 2 - 6 } & 50 & 14.18 & 26.35 & 26.36 & $\mathbf{2 6 . 4 6}$ \\
\hline
\end{tabular}


We have used two different standard images for comparison of PSNR on different noise levels. It is clear from image "Einstein" that at noise level 10, PSNR of noisy image is 28.13. when we have used only TVF then it was increased to 32.34 and when we have used only WBF then it was increased to 33.17 but after combination of TVF and WBF, it is clearly seen that it has now become 33.28 so image denoising is significantly improved. It can also seen that different noise levels, noisy images have different PSNR value and it can be seen that combination of TVF and WBF image denoising method has significant improvement as compared to individual methods.

The process was repeated with another image named "House" and it can be seen that for combination of TVF and WBF image denoising method improved then individual TVF and WBF methods.

\section{Summary and Conclusions}

This paper encompasses implementation of combination of total variation filter (TVF) and weighted bilateral filter (WBF). Detail of both filters is provided along with sufficient mathematical description. The proposed algorithm is applied on images from different standard data sets. From the experimental results, it can be concluded that the proposed algorithm results in a better quality image, both numerically and visually. We have used standards images for our experiments and our results show that the proposed algorithm works well for these images. Furthermore, the experiments are conducted with different noise levels $(10,20,30,40$ and 50).

The image denoising results are compared quantitatively in terms of PSNR and it can be seen that combination of total variation filter (TVF) and weighted bilateral filter (WBF) results in an improved image as compare to only total variation filter (TVF) or only weighted bilateral filter (WBF). Briefly, denoised images of proposed algorithm shows:

- $\quad$ Better reconstruction of sharp edges.

- Better reconstruction of repeating patterns.

- Better preservation of contrast.

- $\quad$ PSNR results are better than WBF for most of the noise levels.

\section{Future Work}

As future research, the proposed scheme can be used for real-time image denoising. In addition neural networks may be used to select the weights of filter for enhanced performance.

\section{Acknowledgments}

The authors would like to thank the anonymous reviewers for their insightful comments and constructive suggestions.

\section{References}

[1] X. Wang and J. Gao. "Image denoising method based on nonsubsampled contourlet transform and bandelet transform", 2009 first international conference on information science and engineering, (2009), pp. 1278-1281.

[2] P. B. Alisha and K. Gnana Sheela, "Image denoising techniques an overview", IOSR journal of electronics and communication engineering (IOSR-JECE), vol. 11, Issue 1, Ver. I, (2016), pp. 78-84.

[3] V. Maheshkar, S. Kamble, S. Agarwal and V. Kumar Srivastava, "Feature image generation using low, mid and high frequency regions for face recognition", The international journal of multimedia \& its applications (IJMA), vol. 4, no.1, (2012).

[4] J. Yan, G. Yang and A. Zhang, "A novel inter-scale correlation image denoising method based on dualtree M-band wavelet", 3rd International conference on intelligent system and knowledge engineering, Xiamen, (2008). 
[5] J. Zheng, L. Zhao, X. Li, X. Zhou and J. Li, "GPU based acceleration of the hyperspectral band selection by SNR estimation using wavelet transform", multispectral hyper spectral and ultra spectral remote sensing technology techniques and applications, proceedings of SPIE international conference, The international society for optical engineering, (2014).

[6] G.Y. Chen, T. D. Bui and A. Krzyzak, "Image denoising using neighboring wavelet coefficients", IEEE International conference on acoustics, speech, and signal processing, vol. 12, no. 1, (2005), pp. 99-107.

[7] J. Benesty, J. Chen, Y. Arden Huang and S. Doclo, "Study of the wiener filter for noise reduction", In speech enhancement signals and communication technology (SCT). Springer, Berlin, Heidelberg, print ISBN 978-3-540-24039-6, online ISBN 978-3-540-27489-6, (2005).

[8] S. Khedkar, K. Akant and M. M. Khanapurkar, " Image denoising using wavelet transform", International journal of research in engineering and technology, vol. 5 issue. 4, (2016), pp. 206-212.

[9] H. Yan Jiang, Z. Yu Cheng, Y. Huo, X. J. Zhou and T. Y. Chai, "Research on image denoising methods based on wavelet transform and rolling-ball algorithm", Proceedings of the international conference on wavelet analysis and pattern recognition, Beijing, China, (2007), pp. 1604-1607.

[10] A.A. Bharath and $\mathrm{J}$. Ng, "A steerable complex wavelet construction and its application to image denoising", IEEE transactions on image processing, vol. 14, no. 7, (2005), pp. 948-959.

[11] J. Zhong, and R. Ning, "Image denoising based on wavelets and multifractals for singularity detection", IEEE transactions on image processing, vol. 14, no. 10, (2005), pp. 1435-1447.

[12] H. Choi and R. G. Baraniuk, "Multiple wavelet basis image denoising using besov ball projections," IEEE signal processing letters, vol. 11, no. 9, (2004), pp. 717-720.

[13] Z. Tongzhou, W. Yanli, R. Ying and L. Yalan, "Approach of image denoising based on discrete multiwavelet transform", International workshop on Intelligent systems and applications, (2009), pp. 1-4.

[14] H. Y. Jiang, Z. Yu Cheng, Y. Huo, X. Jie Zhou and T.Y. Chai, "Research on image denoising methods based on wavelet transform and rolling-ball algorithm", Proceedings of the international conference on wavelet analysis and pattern recognition, Beijing, China, (2007), pp. 1604-1607.

[15] A. Buades, B. Coll and J. Morel, "Nonlocal image and movie denoising". International journal of computer vision, vol. 76, No. 2, (2008), pp. 123-139.

[16] M. Mahmoudi and G. Sapiro, "Fast image and video denoising via nonlocal means of similar neighborhoods", IEEE signal processing Letters, (2005), vol. 12, No. 12, pp. 839-842.

[17] M. Haider, M. Usman, I. Touqir and A. Masood, "Denoising in wavelet domain using probabilistic graphical models", International journal of advanced computer science and applications (IJACSA), vol. 7, issue 11, (2016), pp. 317-321.

[18] S. Peng and L. Lucke, "Multi-level adaptive fuzzy filter for mixed noise removal" Circuits and systems, ISCAS '95, (1995), pp. 1524-1527.

[19] C. Tomasi and R. Manduchi, "Bilateral filtering for gray and color images", Computer vision, 1998. sixth international conference on. (1998), pp.839-846.

[20] B. Zhang and J. P. Allebach, "Adaptive bilateral filter for sharpness enhancement and noise removal", IEEE transactions on image processing, vol.17, Issue 5, (2008), pp. 664- 678.

[21] R. Garnett, T. Huegerich, C. Chui and W. He, "A universal noise removal algorithm with an impulse detector", IEEE transactions on image processing, vol. 14, Issue 11, (2005), pp. 1747-1754.

[22] C. Shen and Z. Lu, "Weighted Bilateral Filter - A new image filter for noise removing and detail information preserving”, 2012 IEEE global high tech congress on electronics (GHTCE), (2012), pp. 1-5.

\section{Authors}

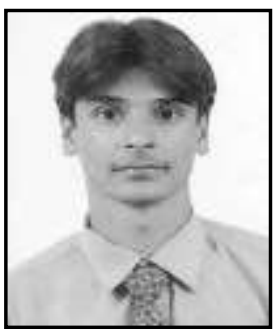

Rashid Ali, he is a $\mathrm{PhD}$ student in the Department of Communication and Information Engineering at the School of Computer \& Communication Engineering, University of Science and Technology Beijing (USTB), Beijing, China. His research interest broadly covers the areas of image processing, Image denoising, Image enhancement. He has published one book in Lambert Academic Publishing Germany.

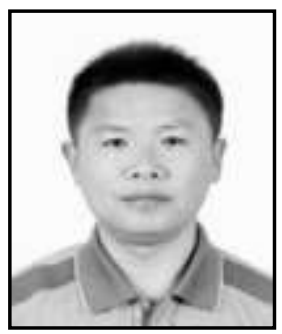

Peng Yunfeng, he is a full time professor in Department of Communication and Information Engineering at the School of Computer \& Communication Engineering, University of Science and Technology Beijing (USTB), Beijing, China. His research interest broadly covers the areas of Image processing, optical internet theory and technology. He has several publication in international conference and ISI, Scopus indexed journals. 


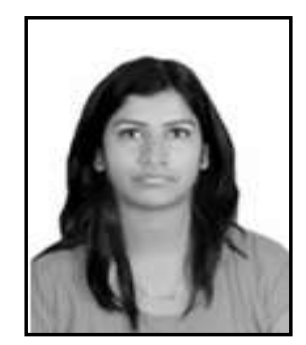

Suman Kumari Gupta, she is a full time lecturer in Department of Information Technology at the School of Computer Networking \& IT Security, Islington College (London Metropolitan University), Kathmandu, Nepal. Her research interest are in Control Engineering, Image Processing and Behavioral Biometrics. 